\title{
Optimization of the Determinant of the Vandermonde Matrix and Related Matrices
}

\author{
Karl Lundengård ${ }^{1} \cdot$ Jonas Österberg ${ }^{1}$ (D) . \\ Sergei Silvestrov ${ }^{1}$
}

Received: 30 October 2015 / Revised: 1 September 2017 /

Accepted: 28 September 2017 / Published online: 14 November 2017

(C) The Author(s) 2017. This article is an open access publication

\begin{abstract}
The value of the Vandermonde determinant is optimized over various surfaces, including the sphere, ellipsoid and torus. Lagrange multipliers are used to find a system of polynomial equations which give the local extreme points in its solutions. Using Gröbner basis and other techniques the extreme points are given either explicitly or as roots of polynomials in one variable. The behavior of the Vandermonde determinant is also presented visually in some interesting cases.
\end{abstract}

Keywords Vandermonde determinant · Optimization · Gröbner basis · Orthogonal polynomials $\cdot$ Ellipsoid $\cdot$ Optimal experiment design $\cdot$ Homogeneous polynomials

Mathematics Subject Classification (2010) 33C45 - 11C20 · 15B99 08 B99

\section{Introduction}

In this paper we will consider the extreme points of the Vandermonde determinant on various surfaces. The examination is primarily motivated by mathematical curiosity but the techniques used here are likely to be extensible to some problems related to optimal experiment design for polynomial regression, see Section 3.

Karl Lundengård

karl.lundengard@mdh.se

Jonas Österberg

jonas.osterberg@mdh.se

Sergei Silvestrov

sergei.silvestrov@mdh.se

1 Division of Applied Mathematics, UKK, Mälardalen University, Högskoleplan 1, Box 883,

72123 Västerås, Sweden 
To the authors knowledge this problem has previously been examined on cubes (see Section 3) and spheres, see Szegő (1939). Here we will consider some techniques to extend some of these results to other surfaces such as ellipsoids, cylinders, $p$-norm spheres and other surfaces defined by homogeneous polynomials. Our examination will mostly be restricted to three dimensions but many of the techniques can in principle be extended to higher dimensions.

\section{The Vandermonde Matrix}

A rectangular Vandermonde matrix of size $m \times n$ is determined by $n$ values $\mathbf{x}=\left(x_{1}, \cdots, x_{n}\right)$ and is defined by

$$
V_{m n}(\mathbf{x})=\left[x_{j}^{i-1}\right]_{m n}=\left[\begin{array}{llll}
1 & 1 & \cdots & 1 \\
x_{1} & x_{2} & \cdots & x_{n} \\
\vdots & \vdots & \ddots & \vdots \\
x_{1}^{m-1} & x_{2}^{m-1} & \cdots & x_{n}^{m-1}
\end{array}\right] .
$$

Note that some authors use the transpose of this as the definition and possibly also let indices run from 0 . All entries in the first row of Vandermonde matrices are ones and by considering $0^{0}=1$ this is true even when some $x_{j}$ is zero. In this paper we will primarily consider square Vandermonde matrices and for convenience we will use the notation $V_{n}(\mathbf{x})=V_{n n}(\mathbf{x})$.

The determinant of the Vandermonde matrix is well known.

Theorem 1 The determinant of square Vandermonde matrices has the form

$$
\operatorname{det} V_{n}(\mathbf{x}) \equiv v_{n}(\mathbf{x})=\prod_{1 \leq i<j \leq n}\left(x_{j}-x_{i}\right)
$$

This determinant is also simply referred to as the Vandermonde determinant or Vandermonde polynomial or Vandermondian (Vein and Dale 1999).

In this paper we will use the method of Lagrange multipliers to optimize the Vandermonde determinant over a surface. For this purpose the following properties will be useful.

Lemma 1 The Vandermonde determinant is a homogeneous polynomial of degree $\frac{n(n-1)}{2}$.

Proof Considering (2) the numbers of factor of $v_{n}(\mathbf{x})$ is $\sum_{i=1}^{n} i-1=\frac{n(n-1)}{2}$. Thus

$$
v_{n}(c \mathbf{x})=c^{\frac{n(n-1)}{2}} v_{n}(\mathbf{x}) .
$$

\section{Application to D-optimal Experiment Designs for Polynomial Regression with a Cost-function}

Suppose an experiment is conducted where $m$ data points from some compact interval, $\mathcal{X} \subset \mathbb{R}, i=1,2, \ldots, m$, are used to create a polynomial regression model of degree 
$n-1$. A vector containing the data points, $\mathbf{x}_{m}=\left(x_{1}, x_{2}, \ldots, x_{m}\right) \in \mathcal{X}^{m}$, is called a design and a design is said to be $D$-optimal if $\operatorname{det}\left(M_{n}\left(\mathbf{x}_{m}\right)\right) \geq \operatorname{det}\left(M_{n}\left(\mathbf{y}_{m}\right)\right)$ for all $\mathbf{y} \in \mathcal{X}^{m}$ where

$$
M_{m}(\mathbf{x})=\left[\begin{array}{llll}
m & \sum_{i=1}^{m} x_{i} & \ldots & \sum_{i=1}^{m} x_{i}^{n-1} \\
\sum_{i=1}^{m} x_{i} & \sum_{i=1}^{m} x_{i}^{2} & \ldots & \sum_{i=1}^{m} x_{i}^{n} \\
\vdots & \vdots & \ddots & \vdots \\
\sum_{i=1}^{m} x_{i}^{n-1} & \sum_{i=1}^{m} x_{i}^{n} & \ldots & \sum_{i=1}^{m} x_{i}^{2 n-2}
\end{array}\right]
$$

is the Fischer information matrix, see Kiefer (1959) and Gaffke and Krafft (1982).

Optimal experiment design is often used to find the minimum number of points needed for a certain model. If we let $m=n$ we get a interpolation problem defined by a square Vandermonde matrix and the Fischer information matrix is $M_{n}(\mathbf{x})=V_{n}(\mathbf{x})^{\top} V_{n}(\mathbf{x})$ and since $V_{n}(\mathbf{x})$ is an $n \times n$ matrix $\operatorname{det}\left(M_{n}(\mathbf{x})\right)=\operatorname{det}\left(V_{n}(\mathbf{x})^{\top}\right) \operatorname{det}\left(V_{n}(\mathbf{x})\right)=\operatorname{det}\left(V_{n}(\mathbf{x})\right)^{2}$. Thus the maximization of the determinant of the Fischer information matrix is equivalent to finding the extreme points of the determinant of a square Vandermonde matrix in some volume given by the set of possible designs, see Gaffke and Krafft (1982).

Optimal designs for various kinds of polynomial regression models are known, see Dette and Trampisch (2010) for an overview. The typical set of possible design is given by constraining each parameter of the model to be in a certain interval. Usually these intervals are also normalized such that the vector of parameters $\mathbf{x}$ can be found in the $n$-dimensional cube $\mathbf{x} \in[-1,1]^{n}$. When consider certain other sets of possible designs the results presented in this paper might be useful.

Suppose there is a cost-function associated with the data such that the total cost of the experiment being below some threshold value, $g(\mathbf{x}) \leq 1$, defines some compact set, $\mathcal{G}=\left\{\mathbf{x} \in \mathbb{R}^{m} \mid g(\mathbf{x}) \leq 1\right\}$, such that $\mathcal{G} \subset \mathcal{X}^{m}$. Since the Vandermonde determinant is a homogeneous polynomial for any $c>1\left|v_{n}(\mathbf{x})\right|>\left|v_{n}(c \mathbf{x})\right|$ the extreme points will be on the surface of the compact set and thus it is enough to consider the set of points defined by $g(\mathbf{x})=1$.

\section{Optimization using Gröbner Bases}

Gröbner bases together with algorithms to find them, and algorithms for solving a polynomial equation is an important tool that arises in many applications. One such application is the optimization of polynomials over affine varieties through the method of Lagrange multipliers. We will here give some main points and informal discussion on these methods as an introduction and to fix some notation.

Definition 1 (Cox et al. 1997) Let $f_{1}, \cdots, f_{m}$ be polynomials in $\mathbb{R}\left[x_{1}, \cdots, x_{n}\right]$. The affine variety $V\left(f_{1}, \cdots, f_{m}\right)$ defined by $f_{1}, \cdots, f_{m}$ is the set of all points $\left(x_{1}, \cdots, x_{n}\right) \in \mathbb{R}^{n}$ such that $f_{i}\left(x_{1}, \cdots, x_{n}\right)=0$ for all $1 \leq i \leq m$. 
When $n=3$ we will sometimes use the variables $x, y, z$ instead of $x_{1}, x_{2}, x_{3}$. Affine varieties are the common zeros of a set of multivariate polynomials. Such sets of polynomials will generate a greater set of polynomials (Cox et al. 1997) by

$$
\left\langle f_{1}, \cdots, f_{m}\right\rangle \equiv\left\{\sum_{i=1}^{m} h_{i} f_{i}: h_{1}, \cdots, h_{m} \in \mathbb{R}\left[x_{1}, \cdots, x_{n}\right]\right\},
$$

and this larger set will define the same variety. But it will also define an ideal (a set of polynomials that contains the zero-polynomial and is closed under addition, and absorbs multiplication by any other polynomial) by $I\left(f_{1}, \cdots, f_{m}\right)=\left\langle f_{1}, \cdots, f_{m}\right\rangle$. A Gröbner basis for this ideal is then a finite set of polynomials $\left\{g_{1}, \cdots, g_{k}\right\}$ such that the ideal generated by the leading terms of the polynomials $g_{1}, \cdots, g_{k}$ is the same ideal as that generated by all the leading terms of polynomials in $I=\left\langle f_{1}, \cdots, f_{m}\right\rangle$.

In this paper we consider the optimization of the Vandermonde determinant $v_{n}(\mathbf{x})$ over surfaces defined by a polynomial equation on the form

$$
s_{n}\left(x_{1}, \cdots, x_{n} ; p ; a_{1}, \cdots, a_{n}\right) \equiv \sum_{i=1}^{n} a_{i}\left|x_{i}\right|^{p}=1,
$$

where we will select the constants $a_{i}$ and $p$ to get ellipsoids in three dimensions, cylinders in three dimensions, and spheres under the $p$-norm in $n$ dimensions. The case of the ellipsoid is suitable for solution by Gröbner basis methods, but due to the existing symmetries the spheres are more suitable for other methods, as provided in Section 8.

From (3) and the convexity of the interior of the sets defined by (4), under a suitable choice of the constant $p$ and non-negative $a_{i}$, it is easy to see that the optimal value of $v_{n}$ on $\sum_{i=1}^{n} a_{i}\left|x_{i}\right|^{p} \leq 1$ will be attained on $\sum_{i=1}^{n} a_{i}\left|x_{i}\right|^{p}=1$. And so, by the method of Lagrange multipliers we have that the minimal/maximal values of $v_{n}\left(x_{1}, \cdots, x_{n}\right)$ on $s_{n}\left(x_{1}, \cdots, x_{n}\right) \leq 1$ will be attained at points such that $\partial v_{n} / \partial x_{i}-\lambda \partial s_{n} / \partial x_{i}=0$ for $1 \leq$ $i \leq n$ and some constant $\lambda$ and $s_{n}\left(x_{1}, \cdots, x_{n}\right)-1=0$, Tyrrell Rockafellar (1993).

For $p=2$ the resulting set of equations will form a set of polynomials in $\lambda, x_{1}, \cdots, x_{n}$. These polynomials will define an ideal over $\mathbb{R}\left[\lambda, x_{1}, \cdots, x_{n}\right]$, and by finding a Grbner basis for this ideal we can use the especially nice properties of Grbner bases to find analytical solutions to these problems, that is, to find roots for the polynomials in the computed basis.

\section{Extreme Points on the Ellipsoid in Three Dimensions}

In this section we will find the extreme points of the Vandermonde determinant on the three dimensional ellipsoid given by

$$
a x^{2}+b y^{2}+c z^{2}=1,(x, y, z) \in \mathbb{R}^{3}
$$

where $a>0, b>0, c>0$.

Using the method of Lagrange multipliers together with (5) and some rewriting gives that all stationary points of the Vandermonde determinant lie in the variety

$$
\begin{aligned}
V= & V\left(a x^{2}+b y^{2}+c z^{2}-1, a x+b y+c z,\right. \\
& a x(z-x)(y-x)-b y(z-y)(y-x)+c z(z-y)(z-x)) .
\end{aligned}
$$


Computing a Gröbner basis for $V$ using the lexicographic order $x>y>z$ give the following three basis polynomials:

$$
\begin{aligned}
g_{1}(z)= & (a+b)(a-b)^{2} \\
& -\left(4(a+b)^{2}(a+c)(b+c)+3 c^{2}\left(a^{2}+a b+b^{2}\right)+3 c\left(a^{3}+b^{3}\right)\right) z^{2} \\
& +3 c(a+b+c)\left(4(a+b)(a+c)(b+c)+\left(a^{2}+b^{2}\right) c+(a+b) c^{2}\right) z^{4} \\
& -c^{2}(b+c)(a+c)(a+b+c)^{2} z^{6}, \\
g_{2}(y, z)= & \left(2(a+b)^{2}(a+c)(b+c)+c\left(a^{2}+2 b^{2}\right)(a+b+c)+2 b c^{2}(a+b)\right) z \\
& +q_{1} z^{5}-q_{2} z^{3}-b(a-b)(a+b)(a+b+3 c) y, \\
g_{3}(x, z)= & \left(2(a+b)^{2}(a+c)(b+c)+c\left(2 a^{2}+b^{2}\right)(a+b+c)+2 a c^{2}(a+b)\right) z \\
& -q_{1} z^{5}+q_{2} z^{3}-a(a-b)(a+b)(a+b+3 c) x, \\
q_{1}= & 9 c^{2}(b+c)(a+c)(a+b+c)^{2}, \\
q_{2}= & 3 c(a+b+c)\left(3 a^{2} b+4 a^{2} c+3 a b^{2}+6 a b c+4 a c^{2}+4 b^{2} c+4 b c^{2}\right) .
\end{aligned}
$$

The calculation of this basis was done using software for symbolic compupation (Maple 18.02 2015).

Since $g_{1}$ only depends on $z$ and $g_{2}$ and $g_{3}$ are first degree polynomial in $y$ and $x$ respectively the stationary points can be found by finding the roots of $g_{1}$ and then calculate the corresponding $x$ and $y$ coordinates. A general formula can be found in this case (since $g_{1}$ only contains even powers of $z$ it can be treated as a third degree polynomial) but it is quite cumbersome and we will therefore not give it explicitly.

Note that in general the polynomials in the Gröbner basis are not guaranteed to have real-valued roots. For the ellipsoid case examined here it can be shown that the computed Gröbner basis will only give real-valued coordinates. Since the extreme points of the Vandermonde determinant is a subset of the points with coordinates given by the roots of the polynomials in the Gröbner basis this means that we will not need to check that the found points are real-valued or not, we only need to check whether they are proper extreme points or not.

Lemma 2 The polynomial equation system defined by (6)-(8) will only have solutions with real-valued $x, y$ and $z$.

Proof The discriminant is a useful tool for determining how many real roots low-level polynomials have. Following Irving (2004) the discriminant, $\Delta(p)$, of a third degree polynomial $p(x)=c_{0}+c_{1} x+c_{2} x^{2}+c_{3} x^{3}$ is

$$
\Delta(p)=18 c_{1} c_{2} c_{3} c_{4}-4 c_{2}^{3} c_{4}+c_{2}^{2} c_{3}^{2}-4 c_{1} c_{3}^{3}-27 c_{1}^{2} c_{4}^{2}
$$

and if $\Delta(p)$ is non-negative then all roots will be real (but not necessarily distinct). Since the first basis polynomial $g_{1}$ only contains terms with even exponents and is of degree 6 the polynomial $\tilde{g}_{1}$ defined by $\tilde{g}_{1}\left(z^{2}\right)=g_{1}(z)$ will be a polynomial of degree 3 whose roots are the square fo the roots of $g_{1}$. Calculating the discriminant of $\tilde{g}_{1}$ gives

$$
\begin{aligned}
\Delta\left(\tilde{g}_{1}\right)= & 9(a-b)^{2}(a+b+3 c)^{2}(a+b+c)^{4} a b c^{3} \\
& \left(32\left(a^{3} b^{2}+a^{3} c^{2}+a^{2} b^{3}+a^{2} c^{3}+b^{3} c^{2}+b^{2} c^{3}\right)+61 a b c(a+b+c)^{2}\right) .
\end{aligned}
$$


Since $a, b$ and $c$ are all positive numbers it is clear that $\Delta\left(g_{1}\right)$ is non-negative. Furthermore, since $a, b$ and $c$ are positive numbers all terms in $\tilde{g}_{1}$ with odd powers have negative coefficients and all terms with even powers have positive coefficients. Thus if $w<0$ then $\tilde{g}_{1}(w)>0$ and thus all roots must be positive. Since $g_{2}$ is a first order polynomial with respect to $y$ a real-valued $z$ will give a real-valued $y$. Since $g_{3}$ is a first order polynomial with respect to $x$ a real-valued $z$ will give a real-valued $x$.

An illustration of an ellipsoid and the extreme points of the Vandermonde determinant on its surface is shown in Fig. 1.

\section{Extreme Points on the Cylinder in Three Dimensions}

In this section we will examine the local extreme points on an infinitely long in 3 dimensions cylinder aligned with the $x$-axis. In this case we do not need to use Gröbner basis techniques since the problem can be reduced to a one dimensional polynomial equation.

Consider the cylinder defined by

$$
b y^{2}+c z^{2}=1 \text {, where } b>0, c>0 .
$$

Using the method of Lagrange multipliers gives the equation system

$$
\begin{aligned}
& \frac{\partial v_{3}}{\partial x}=0, \\
& \frac{\partial v_{3}}{\partial y}=2 \lambda b y, \\
& \frac{\partial v_{3}}{\partial z}=2 \lambda c z .
\end{aligned}
$$

Taking the sum of each expression gives

$$
b y+c z=0 \Leftrightarrow y=-\frac{c}{b} z .
$$

Combining (9) and (10) gives

$$
\left(\frac{c}{b}+1\right) c z^{2}=1 \Rightarrow z= \pm \sqrt{\frac{b}{c}} \frac{1}{\sqrt{b+c}} \Rightarrow y=\mp \sqrt{\frac{c}{b}} \frac{1}{\sqrt{b+c}} .
$$
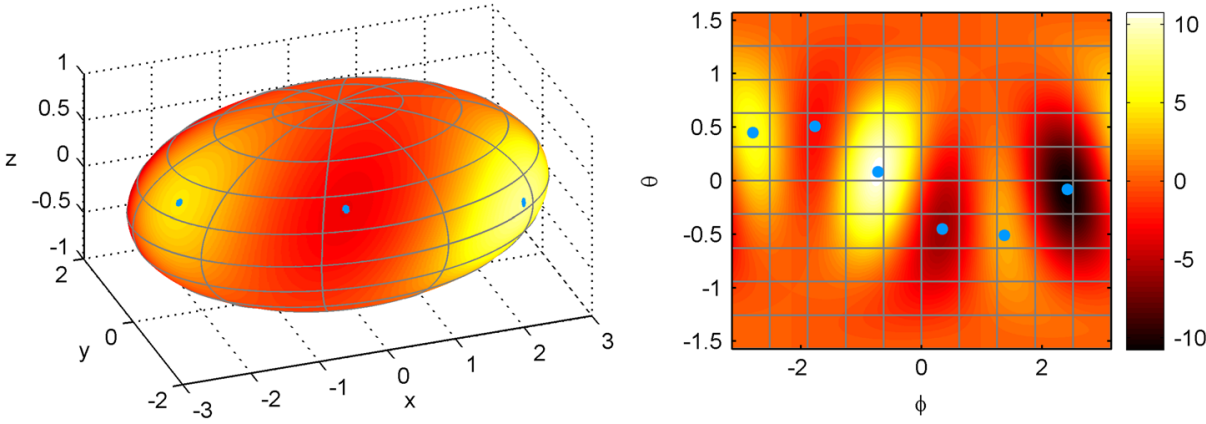

Fig. 1 Illustration of the ellipsoid defined by $\frac{x^{2}}{9}+\frac{y^{2}}{4}+z^{2}=0$ with the extreme points of the Vandermonde determinant marked. Displayed in Cartesian coordinates on the right and in ellipsoidal coordinates on the left 
Thus the plane defined by (10) intersects with the cylinder along the lines

$$
\begin{aligned}
& \ell_{1}=\left\{\left(x, \sqrt{\frac{c}{b}} \frac{1}{\sqrt{b+c}},-\sqrt{\frac{b}{c}} \frac{1}{\sqrt{b+c}}\right) \mid x \in \mathbb{R}\right\}=\{(x, r,-s) \mid x \in \mathbb{R}\}, \\
& \ell_{2}=\left\{\left(x,-\sqrt{\frac{c}{b}} \frac{1}{\sqrt{b+c}}, \sqrt{\frac{b}{c}} \frac{1}{\sqrt{b+c}}\right) \mid x \in \mathbb{R}\right\}=\{(x,-r, s) \mid x \in \mathbb{R}\} .
\end{aligned}
$$

Finding the stationary points for $v_{3}$ along $\ell_{1}$ :

$$
\begin{aligned}
v_{3}(x, r,-s) & =\left(x^{2}+\frac{1}{\sqrt{b+c}}\left(\sqrt{\frac{b}{c}}-\sqrt{\frac{c}{b}}\right) x+\frac{1}{b+c}\right)(r+s), \\
\frac{\partial v_{3}}{\partial x}(x, r,-s) & =\left(2 x+\frac{1}{\sqrt{b+c}}\left(\sqrt{\frac{b}{c}}-\sqrt{\frac{c}{b}}\right)\right)(r+s) .
\end{aligned}
$$

From this it follows that

$$
\frac{\partial v_{3}}{\partial x}(x, r,-s)=0 \Leftrightarrow x=\frac{1}{2 \sqrt{b+c}}\left(\sqrt{\frac{c}{b}}-\sqrt{\frac{b}{c}}\right) .
$$

Thus

$$
\mathbf{x}_{1}=\frac{1}{\sqrt{b+c}}\left(\frac{1}{2}\left(\sqrt{\frac{c}{b}}-\sqrt{\frac{b}{c}}\right), \sqrt{\frac{c}{b}},-\sqrt{\frac{b}{c}}\right)
$$

is the only stationary point on $\ell_{1}$. An analogous argument shows that $\mathbf{x}_{2}=-\mathbf{x}_{1}$ is the only stationary point on $\ell_{2}$.

An example of where these points are placed on the cylinder is shown in Fig. 2.

\section{Optimizing the Vandermonde Determinant on a Surface Defined by a Homogeneous Polynomial}

When using Lagrange multipliers it can be desirable to not have to consider the $\lambda$-parameter (the scaling between the gradient and direction given by the constraint). we demonstrate a simple way to remove this parameter when the surface is defined by an homogeneous polynomial.
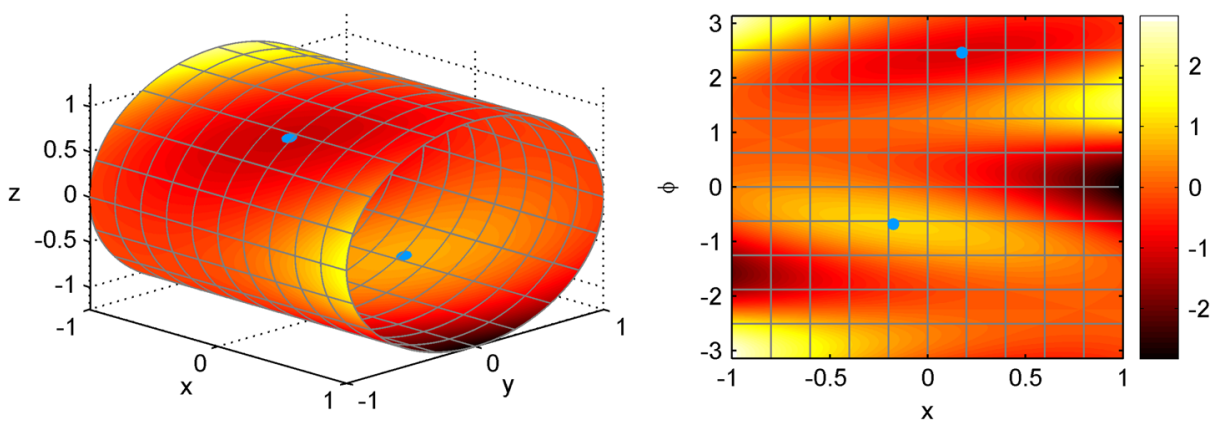

Fig. 2 Illustration of the cylinder defined by $y^{2}+\frac{16}{25} z^{2}=1$ with the extreme points of the Vandermonde determinant marked. Displayed in Cartesian coordinates on the right and in cylindrical coordinates on the left 
Lemma 3 Let $g: \mathbb{R}^{n} \rightarrow \mathbb{R}^{n}$ be a homogeneous polynomial such that $g(c \mathbf{x})=c^{k} g(\mathbf{x})$ with $k \neq \frac{n(n-1)}{2}$ such that $g(\mathbf{x})=1, \mathbf{x} \in \mathbb{R}^{n}$ defines a continuous bounded surface. Let $\mathbf{z} \in \mathbb{R}^{n}$ be a point on the surface. Then $\mathbf{z}$ can be written as $\mathbf{z}=c \mathbf{y}$ where

$$
\left.\frac{\partial v_{n}}{\partial x_{i}}\right|_{\mathbf{x}=\mathbf{y}}=\left.\frac{\partial g}{\partial x_{i}}\right|_{\mathbf{x}=\mathbf{y}}, i \in 1,2, \ldots, n
$$

and $c=g(\mathbf{y})^{-\frac{1}{k}}$, if and only if $\mathbf{z}$ a stationary point for the Vandermonde determinant.

Proof By the method of Lagrange multipliers the point $\mathbf{y} \in\left\{\mathbf{x} \in \mathbb{R}^{n} \mid g(\mathbf{x})=1\right\}$ is a stationary point for the Vandermonde determinant and only if

$$
\left.\frac{\partial v_{n}}{\partial x_{k}}\right|_{\mathbf{x}=\mathbf{y}}=\left.\lambda \frac{\partial g}{\partial x_{k}}\right|_{\mathbf{x}=\mathbf{y}}, k \in 1,2, \ldots, n
$$

for some $\lambda \in \mathbb{R}$.

The stationary points on the surface given by $g(c \mathbf{x})=c^{k}$ will be given by

$$
\left.c^{\frac{n(n-1)}{2}} \frac{\partial v_{n}}{\partial x_{k}}\right|_{\mathbf{x}=\mathbf{y}}=\left.c^{k} \lambda \frac{\partial g}{\partial x_{k}}\right|_{\mathbf{x}=\mathbf{y}}, k \in 1,2, \ldots, n
$$

and if $c$ is chosen such that $\lambda=c^{\frac{n(1-n)}{2}} c^{k}$ then the stationary points are defined by

$$
\frac{\partial v_{n}}{\partial x_{k}}=\frac{\partial g}{\partial x_{k}}, \quad k \in 1,2, \ldots, n .
$$

Suppose that $\mathbf{y} \in\left\{\mathbf{x} \in \mathbb{R}^{n} \mid g(\mathbf{x})=c^{k}\right\}$ is a stationary point for $v_{n}$ then the point given by $\mathbf{z}=c \mathbf{y}$ where $c=g(\mathbf{y})^{-\frac{1}{k}}$ will be a stationary point for the Vandermonde determinant and will lie on the surface defined by $g(\mathbf{x})=1$.

Lemma 4 If $\mathbf{z}$ is a stationary point for the Vandermonde determinant on the surface $g(\mathbf{x})=$ 1 where $g(\mathbf{x})$ is a homogeneous polynomial then $-\mathbf{z}$ is either a stationary point or does not lie on the surface.

Proof Since $g(-\mathbf{x})=(-1)^{k} g(\mathbf{x})$ is either 1 or -1 then $\left|v_{n}(\mathbf{x})\right|=\left|v_{n}(-\mathbf{x})\right|$ for any point, including $\mathbf{z}$ and the points in a neighbourhood around it which means that if $g(-\mathbf{x})=$ $g(\mathbf{x})$ then the stationary points are preserved and otherwise the point will lie on the surface defined by $g(\mathbf{x})=-1$ instead of $g(\mathbf{x})=1$.

A well-known example of homogeneous polynomials are quadratic forms. If we let

$$
g(\mathbf{x})=\mathbf{x}^{\top} S \mathbf{x}
$$

then $g(\mathbf{x})$ is a quadratic form which in turn is a homogeneous polynomial with $k=2$. If $S$ is a positive definite matrix then $g(\mathbf{x})=1$ defines an ellipsoid. Here will will demonstrate the use of Lemma 3 to find the extreme points on a rotated ellipsoid.

Consider the ellipsoid defined by

$$
\frac{1}{9} x^{2}+\frac{5}{8} y^{2}+\frac{3}{4} y z+\frac{5}{8} z^{2}=1
$$


then by Lemma 3 we can instead consider the points in the variety

$$
\begin{aligned}
V= & V\left(-2 x y+2 x z+y^{2}-z^{2}-\frac{2}{9} x,\right. \\
& -x^{2}+2 x y-2 y z+z^{2}-\frac{5}{4} y-\frac{3}{4} z, \\
& \left.-2 x z-y^{2}+2 y z+x^{2}-\frac{3}{4} y-\frac{5}{4} z\right) .
\end{aligned}
$$

Finding the Gröbner basis of $V$ gives

$$
\begin{aligned}
g_{1}(z) & =z(6 z+1)\left(260642 z^{2}-27436 z+697\right), \\
g_{2}(y, z) & =-1138484256 z^{3}-127275604 z^{2}+16689841 z+6277879 y, \\
g_{3}(x, z) & =10246358304 z^{3}+1145480436 z^{2}-93707658 z+6277879 x .
\end{aligned}
$$

This system is not difficult to solve and the resulting points are:

$$
\begin{aligned}
& p_{0}=(0,0,0), \\
& p_{1}=\left(0, \frac{1}{6},-\frac{1}{6}\right), \\
& p_{2}=\left(\frac{45 \sqrt{2}}{361},-\frac{1}{19}-\frac{5 \sqrt{2}}{722}, \frac{1}{19}-\frac{5 \sqrt{2}}{722}\right), \\
& p_{3}=\left(\frac{45 \sqrt{2}}{361},-\frac{1}{19}+\frac{5 \sqrt{2}}{722}, \frac{1}{19}+\frac{5 \sqrt{2}}{722}\right) .
\end{aligned}
$$

The point $p_{0}$ is not a valid solution since it does and does not lie on any ellipsoid defined by (5) and can therefore be discarded. By Lemma 4 there are also three more stationary points $p_{4}=-p_{1}, p_{5}=-p_{2}$ and $p_{6}=-p_{3}$. Rescaling each of these points according to Lemma 3 gives $q_{i}=\sqrt{g\left(p_{i}\right)}$ which are all points on the ellipsoid defined by $g(\mathbf{x})=1$. The result is illustrated in Fig. 3.

Note that this example gives a simple case with a small Gröbner basis that is small and easy to find. Using this technique for other polynomials and in higher dimensions can require significant computational resources.
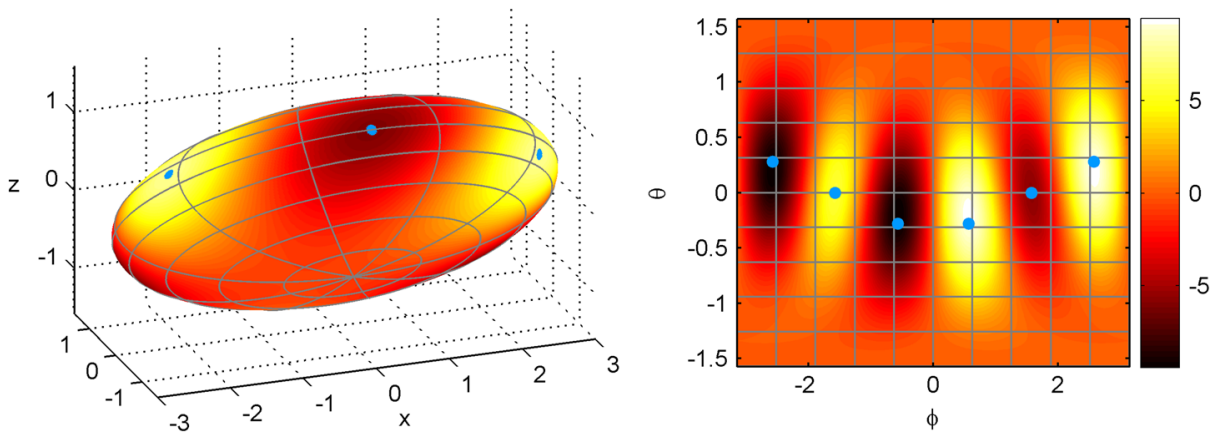

Fig. 3 Illustration of the ellipsoid defined by (13) with the extreme points of the Vandermonde determinant marked. Displayed in Cartesian coordinates on the right and in ellipsoidal coordinates on the left 


\section{The Vandermonde Determinant on $p$-norm Spheres}

The optimization of the Vandermonde determinant on the sphere $(p=2)$ and on the cube $(p=\infty)$ lends themselves to methods in orthogonal polynomials. In fact, as shown by Stieltjes and recaptured by Szegő (1939), and presented in more detail in and extended in (Lundengård et al. 2013) there is a fairly straightforward solution derived from electrostatic considerations, which implicitly deals with the Vandermonde determinant.

Consider the optimization of $v_{n}(\mathbf{x})$ over the sphere

$$
s_{n}(\mathbf{x})=\sum_{i=1}^{n} x_{i}^{p}=1,
$$

for suitable choices for $p$ (even). Instead of optimizing $v_{n}$ we are free to optimize $\ln \left|v_{n}\right|$ over the sphere to the same effect $\left(v_{n}(\mathbf{x})=0\right.$ is not a solution, all $x_{i}$ are pair-wise distinct). This leaves us with the set of equations by Lagrange multipliers.

$$
\frac{\partial \ln \left|v_{n}\right|}{\partial x_{k}}=\lambda \frac{\partial s_{n}}{\partial x_{k}} \quad, \quad s_{n}=1,
$$

where the left-most equation holds for $1 \leq k \leq n$. It is easy to show that the partial derivatives can be written

$$
\frac{\partial \ln \left|v_{n}\right|}{\partial x_{k}}=\sum_{\substack{i=1 \\ i \neq k}}^{n} \frac{1}{x_{k}-x_{i}} .
$$

From this it is easy to show that (15) can be rewritten by introducing the univariate polynomial $P_{n}(x)=\prod_{i=1}^{n}\left(x-x_{i}\right)$ as

$$
\frac{\partial \ln \left|v_{n}\right|}{\partial x_{k}}=\frac{1}{2} \frac{P^{\prime \prime}\left(x_{k}\right)}{P^{\prime}\left(x_{k}\right)} .
$$

Now the leftmost equation of (14) can be written

$$
\frac{1}{2} \frac{P^{\prime \prime}\left(x_{k}\right)}{P^{\prime}\left(x_{k}\right)}=\lambda \frac{\partial s_{n}}{\partial x_{k}}
$$

or more succinct

$$
P^{\prime \prime}\left(x_{k}\right)-2 \lambda \frac{\partial s_{n}}{\partial x_{k}} P^{\prime}\left(x_{k}\right)=0,
$$

In the case $p=2$ we are lucky since (16) becomes, by introducing the new "multiplier" $\rho$

$$
\left.\left(P^{\prime \prime}(x)+\rho_{n} x P^{\prime}(x)\right)\right|_{x=x_{k}}=0,
$$

and since the left part of this equation is a polynomial of degree $n$ and has roots $x_{1}, \cdots, x_{n}$ we must have

$$
P^{\prime \prime}(x)+\rho_{n} x P^{\prime}(x)+\sigma_{n} P(x)=0,
$$

for some $\rho_{n}, \sigma_{n}$ that may depend on $n$. Now if choose $P(x)$ to be monic, note that $\rho_{n} \neq 0$, and require us to be on the sphere we get $P(x)=x^{n}-\frac{1}{2} x^{n-2}+\cdots$, and by identifying coefficients we get $\rho_{n}$ and $\sigma_{n}$ :

$$
P^{\prime \prime}(x)+n(1-n) x P^{\prime}(x)+n^{2}(n-1) P(x)=0,
$$

which is a nice and well known form of differential equation and defines a sequence of orthogonal polynomials that are rescaled Hermite polynomials (Szegő 1939), so we can find a recurrence relation for $P_{n+1}$ in terms of $P_{n}$ and $P_{n-1}$, and we can, for a fixed $n$, construct the coefficients of $P_{n}$ recursively, without explicitly finding $P_{1}, \cdots, P_{n-1}$. 
Now, this is for $p=2$. For $p=4$ we continue from (16) instead with

$$
\left.\left(P^{\prime \prime}(x)+\rho_{n} x^{3} P^{\prime}(x)\right)\right|_{x=x_{k}}=0 .
$$

Now the polynomial in $x$ in the left part of this equation has shared roots with $P(x)$ and so by the same method as for $p=2$ we get:

$$
P^{\prime \prime}(x)+\rho_{n} x^{3} P^{\prime}(x)+\left(\sigma_{n} x^{2}+\tau_{n} x+v_{n}\right) P(x)=0 .
$$

It is easy to show for the sphere under any $p$-norm that the extreme points of $\ln \left|v_{n}(\mathbf{x})\right|$ where $x_{1}<\cdots<x_{n}$ are unique, see Szegó (1939) and Lundengård et al. (2013), this coupled with the symmetry relation $\ln \left|v_{n}(\mathbf{x})\right|=\ln \left|v_{n}(-\mathbf{x})\right|$, provides us with the property that the extreme points are symmetric in the sense that for all $1 \leq i \leq n$ we have that there exists a $1 \leq j \leq n$ such that $x_{i}=-x_{j}$, for odd $n$ we then have that $x_{i}=0$ for some $i$. We thus get polynomials $P(x)$ on the form:

$$
P(x)=x^{n}+c_{n-2} x^{n-2}+c_{n-4} x^{n-4}+\cdots,
$$

with every other coefficient zero, for even $n$ we have only even powers, for odd $n$ we have odd powers. By identifying powers in (20) we get that $\tau_{n} x P(x)$ will not share any powers with any other part of the equation and so $\tau_{n}=0$. We can also by identifying coefficients get $n \rho_{n}+\sigma_{n}=0$. We now have

$$
P^{\prime \prime}(x)+\rho_{n} x^{3} P^{\prime}(x)+\left(-n \rho_{n} x^{2}+v_{n}\right) P(x)=0 .
$$

For $n=2$ we get the specific system

$$
2+\rho x^{3}(2 x)+\left(-2 \rho x^{2}+v\right)\left(x^{2}+c_{0}\right)=0,
$$

but we actually don't need to calculate much here since it is easy do adapt the roots of $x^{2}+c_{0}$ to the sphere with $p=4$, we get:

$$
P_{2}^{4}(x)=x^{2}-\frac{1}{\sqrt{2}}
$$

The case $n=3$ is also easy and by symmetry we get a zero coordinate:

$$
P_{3}^{4}(x)=x^{3}-\frac{1}{\sqrt{2}} x .
$$

The case $n=4$ becomes a bit more interesting:

$$
\begin{array}{r}
\left(12 x^{2}+2 c_{2}\right)+\rho x^{3}\left(4 x^{3}+2 c_{2} x\right)+\left(-4 \rho x^{2}+v\right)\left(x^{4}+c_{2} x^{2}+c_{0}\right)=0, \\
\left(v-2 \rho c_{2}\right) x^{4}+\left(12+v c_{2}-4 \rho c_{0}\right) x^{2}+\left(2 c_{2}+v c_{0}\right)=0,
\end{array}
$$

This provides three equations. Now letting $t=x^{2}$ so that

$$
P(t)=t^{2}+c_{2} t+c_{0}=\left(t-t_{1}\right)\left(t-t_{2}\right)=t^{2}-\left(t_{1}+t_{2}\right) t+t_{1} t_{2},
$$

gives us the last equation $\sum x_{i}^{4}=2 \sum t_{i}^{2}=2\left(c_{2}^{2}-2 c_{0}\right)=1$. Solving this gives us

$$
P_{4}^{4}(x)=x^{4}-\frac{2}{\sqrt{6}} x^{2}+\frac{1}{12} \text {. }
$$

\section{Conclusion}

In this paper we have examined the extreme points of the Vandermonde determinant on various surfaces in three dimensions. Explicit expressions for the placement of the extreme 
points on an ellipsoid aligned with the axis and a cylinder aligned with the $x$-axis were found using Gröbner bases in Sections 5 and 6.

A convenient way of rewriting the system of polynomial equations that the method of Lagrange multipliers gives was shown in Section 7 and it was illustrated how this rewrite could be used to find the extreme points on an ellipsoid not aligned with the coordinate system.

In Section 8 a method for finding the extreme points on spheres with $p$-norm was discussed, specifically the case when $p=4$ for two, three and four dimensions.

Further work will be to extend the methods described here to higher dimensions and also examine matrices related to the Vandermonde matrices, for instance the matrices described in Section 3.

Open Access This article is distributed under the terms of the Creative Commons Attribution 4.0 International License (http://creativecommons.org/licenses/by/4.0/), which permits unrestricted use, distribution, and reproduction in any medium, provided you give appropriate credit to the original author(s) and the source, provide a link to the Creative Commons license, and indicate if changes were made.

\section{References}

Cox D, Little J, O'Shea D (1997) Ideals, varieties, and algorithms. Springer, New York

Dette H, Trampisch M (2010) A general approach to D-optimal designs for weighted univariate polynomial regression models. Journal of the Korean Statistical Society 39:1-26

Gaffke N, Krafft O (1982) Exact D-Optimum Designs for Quadratic Regression. J R Stat Soc Ser B Methodol 44(3):394-397

Irving RS (2004) Integers, Polynomials and rings. Undergraduate texts in mathematics. Springer, New York

Kiefer JC (1959) Optimum experimental designs. J R Stat Soc Ser B Methodol 21(2):272-319

Lundengård K, Österberg J, Silvestrov S (2013) Extreme points of the Vandermonde determinant on the sphere and some limits involving the generalized Vandermonde determinant. arXiv:1312.6193

Tyrrell Rockafellar R. (1993) Lagrange multipliers and optimality. SIAM Rev 35(2):183-238

Szegő G (1939) Orthogonal polynomials. American Mathematics Society

Vein R, Dale P (1999) Determinants and their applications in mathematical physics applied mathematical sciences, vol 134. Springer, New York

Maple 18.02 (2015) Maplesoft, a division of Waterloo Maple Inc., Waterloo, Ontario 